\title{
ETosis: A Microbicidal Mechanism beyond Cell Death
}

\author{
Anderson B. Guimarães-Costa, ${ }^{1}$ Michelle T. C. Nascimento, ${ }^{1}$ \\ Amanda B. Wardini, ${ }^{2}$ Lucia H. Pinto-da-Silva, ${ }^{3}$ and Elvira M. Saraiva ${ }^{1}$ \\ ${ }^{1}$ Instituto de Microbiologia Paulo de Góes, Universidade Federal do Rio de Janeiro (UFRJ), 21941-901 Rio de Janeiro, RJ, Brazil \\ ${ }^{2}$ Instituto de Biofísica Carlos Chagas Filho, Universidade Federal do Rio de Janeiro (UFRJ), 21941-901 Rio de Janeiro, RJ, Brazil \\ ${ }^{3}$ Instituto de Veterinária, Universidade Federal Rural do Rio de Janeiro (UFRRJ), 23890-000 Seropedica, RJ, Brazil
}

Correspondence should be addressed to Elvira M. Saraiva, esaraiva@micro.ufrj.br

Received 30 August 2011; Accepted 10 November 2011

Academic Editor: Dario Zamboni

Copyright (c) 2012 Anderson B. Guimarães-Costa et al. This is an open access article distributed under the Creative Commons Attribution License, which permits unrestricted use, distribution, and reproduction in any medium, provided the original work is properly cited.

Netosis is a recently described type of neutrophil death occurring with the release to the extracellular milieu of a lattice composed of DNA associated with histones and granular and cytoplasmic proteins. These webs, initially named neutrophil extracellular traps (NETs), ensnare and kill microorganisms. Similarly, other cell types, such as eosinophils, mast cells, and macrophages, can also dye by this mechanism; thus, it was renamed as ETosis, meaning death with release of extracellular traps (ETs). Here, we review the mechanism of NETosis/etosis, emphasizing its role in diseases caused by protozoan parasites, fungi, and viruses.

\section{Introduction}

Upon inflammation, neutrophils are the first cells to be recruited to the inflammatory site, in a process orchestrated by chemokines, a series of attractive molecules produced locally. Neutrophils then migrate to the inflamed site where they contain and eliminate microorganisms using three basic strategies: phagocytosis, with ingestion and killing of microorganisms inside special compartments of the cell, degranulation, which consists in the extravasation of the granules content to the extracellular milieu, and by a new antimicrobial mechanism named netosis that also occurs in the extracellular milieu, when DNA associated to proteins is expelled from the cell [1-3].

Neutrophils are the most abundant leukocytes in the blood and constitute the first line of host defense against invading pathogens. These cells are also known as polymorphonuclears (PMNs) or granulocytes, since they have a segmented nucleus with lobules linked by nuclear filaments and hold large numbers of three different types of granules. These granules were classified according to their protein content as primary, azurophil, or peroxidase-positive granules because of their high myeloperoxidase (MPO) loading. Besides MPO, these granules possess cathepsin $\mathrm{G}$, defensins, elastase, and proteinase 3, among many others proteins. MPO production stops between promyelocyte and myelocyte transition stages during maturation at the bone marrow, and then, the next granules formed are all peroxidase negative. The secondary granules contain collagenase, gelatinase, lactoferrin, and sialidase, and the tertiary granules enclose gelatinase, $\beta 2$ microglobulin, and others. In addition, secretory vesicles are also present in the neutrophil cytoplasm; however, the protein content of these vesicles has not been completely elucidated $[4,5]$.

\section{NETosis Occurs with Extrusion of Neutrophil Extracellular Traps (NETs)}

In a seminal work, Brinkmann and colleagues in 2004 described that upon activation by phorbol myristate acetate (PMA), lipopolysaccharide (LPS), interleukin 8 (IL-8), or by Gram-positive and -negative bacteria, neutrophils release their chromatin to the extracellular medium, associated with different proteins, forming the so-called NETs. Currently, there is an increasing list of synthetic and physiological molecules, as well as microorganisms and their products, which can activate neutrophils to release NETs (Table 1). 
TABLE 1: Microorganisms or molecules able to trigger extracellular traps release.

\begin{tabular}{|c|c|c|}
\hline Activators & Cells & Reference \\
\hline Activated endothelial cell & Neutrophil & [6] \\
\hline Antiribonucleoprotein IgG & SLE Neutrophil & [7] \\
\hline Aspergillus fumigatus (conidia or hyphae) & Neutrophil & {$[8,9]$} \\
\hline Autoantibodies (anti-LL-37/anti-HNP) & SLE Neutrophil & {$[10]$} \\
\hline Calcium & HL-60 lineage, neutrophil & {$[11,12]$} \\
\hline Candida albicans (hyphae or yeast) & Neutrophil & [13] \\
\hline Cryptococcus gattii & Neutrophil & {$[14]$} \\
\hline Cryptococcus neoformans & Neutrophil & {$[15]$} \\
\hline Eimeria bovis & Neutrophil & {$[16]$} \\
\hline Enterococcus faecalis & Neutrophils & [17] \\
\hline Equine spermatozoa & Neutrophil & {$[18]$} \\
\hline Escherichia coli & Neutrophil, monocyte & {$[17,19-21]$} \\
\hline Glucose oxidase & Neutrophil & [22] \\
\hline $\mathrm{GM}-\mathrm{CSF}+\mathrm{C} 5 \mathrm{a}$ & Neutrophil & {$[23,24]$} \\
\hline GM-CSF + LPS & Neutrophil & {$[24]$} \\
\hline Haemophilus influenzae & Neutrophil & {$[25,26]$} \\
\hline Helicobacter pylori & Neutrophil & [27] \\
\hline Hydrogen peroxide & Neutrophil, mast cell, chicken heterophil & {$[22,28,29]$} \\
\hline Interferon (IFN)- $\alpha+$ C5a & Neutrophil & {$[23]$} \\
\hline IFN- $\gamma+$ C5a & Neutrophil, eosinophil & {$[23,30]$} \\
\hline IFN- $\gamma+$ C5a, LPS or eotaxin & Eosinophil & {$[30]$} \\
\hline Interleukin $5+$ LPS/C5a/eotaxin & Eosinophil & {$[30]$} \\
\hline Interleukin 8 & Neutrophil & {$[1,31]$} \\
\hline Interleukin 23 and IL- $1 \beta$ & Mast cells & {$[32]$} \\
\hline Klebsiella pneumoniae & Neutrophil (tissue) & [33] \\
\hline Lactococcus lactis & Neutrophil & {$[34]$} \\
\hline Leishmania amazonensis (promastigotes/amastigotes/lipophosphoglycan) & Neutrophil & [35] \\
\hline Leishmania amazonensis, L. donovani, L. major, L. chagasi (promastigotes) & Neutrophil & [35-37] \\
\hline Lipopolysaccharide (LPS) & Neutrophil & {$[1]$} \\
\hline Listeria monocytogenes & Neutrophil & {$[20,38]$} \\
\hline Mannheimia haemolytica and leukotoxin & Neutrophil & [39] \\
\hline M1 protein/M1 protein-fibrinogen complex & Neutrophil, mast cell & {$[40,41]$} \\
\hline Mycobacterium tuberculosis, M. canettii & Neutrophil & {$[42]$} \\
\hline Nitric oxide & Neutrophil & {$[43,44]$} \\
\hline Panton-Valentine leukocidin, autolysin, and lipase & Neutrophil & {$[45]$} \\
\hline Phorbol myristate acetate (PMA) & Neutrophil, mast cell, chicken heterophil & {$[1,12,28,29]$} \\
\hline $\mathrm{PMA}+$ ionomycin & Neutrophil & [17] \\
\hline Platelet-activating factor & Neutrophil & [27] \\
\hline Platelet TLR-4 & Neutrophil & {$[46]$} \\
\hline Pseudomonas aeruginosa & Mast cell & [28] \\
\hline Serratia marcescens & Neutrophil & [17] \\
\hline Shigella flexneri & Neutrophil (tissue) & [1] \\
\hline Staphylococcus aureus & Neutrophil, mast cell & {$[22,28,45]$} \\
\hline Statins & Neutrophil, monocytes/macrophages & {$[47]$} \\
\hline Streptococcus (Group A-GAS)/Pilus & Neutrophil, mast cell & {$[34,40,48]$} \\
\hline Streptococcus dysgalactiae & Neutrophil & [17] \\
\hline Streptococcus pneumoniae & Neutrophil (tissue) & [49] \\
\hline Streptococcus pyogenes & Mast cell & [28] \\
\hline Syncytiotrophoblast microparticles & Neutrophil & {$[31]$} \\
\hline
\end{tabular}


TABle 1: Continued.

\begin{tabular}{lcc}
\hline Activators & Cells & Reference \\
\hline TNF- $\alpha$ & HL-60 lineage & {$[11]$} \\
TNF- $\alpha+$ ANCA IgG & Neutrophil & {$[50]$} \\
Yeast particulate B-glucan & Neutrophil & {$[51]$} \\
Yersinia enterocolitica & Neutrophil & {$[52]$} \\
$\delta$-Toxin from Staphylococcus epidermidis & Neutrophil & {$[53]$} \\
\hline
\end{tabular}

Initially, NET composition was described as being formed by decondensed chromatin scaffolds associated to proteins of the different types of neutrophil granules [1]. Nicotinamide adenine dinucleotide phosphate (NADPH) oxidase subunits, human peptidoglycan protein short-S, and pentraxin (PTX)-3 were also detected in association with NETs $[20,54,55]$. Later, using proteomic analysis, besides histones and granule proteins, cytosolic and cytoskeleton proteins, catalase and glycolytic enzymes were found linked to the NETs [15]. This proteomic study evidenced that among the NET-associated proteins, histones were the most abundant, followed by the neutrophil elastase. Intriguingly, NADPH oxidase, PTX3, and cathelicidin LL-37 previously detected in NETs by immunofluorescence staining were not found associated to NETs by proteomic analysis [15]. These discrepancies could be due to a loose association of these missing proteins to the NETs, which could have been lost by the NET processing for the proteomic analysis. Recently, IL17 was found associated to NETs in psoriasis skin biopsies evidenced with specific antibodies by immunofluorescence staining [32].

\section{NETosis Mechanism}

Although very little is known about the mechanism of NET release, some morphological features are easily observed during netosis (Figure 1). Thus, after stimulation, neutrophil nuclei lose segregation of eu- and heterochromatin, its characteristic lobular form vanishes, and the nuclear membrane swells up, fragmenting into vesicles. This is followed by the granules' membranes disruption, which allows the mixing of nuclear, cytoplasmic, and granular contents. Subsequently, the plasma membrane is permeabilized, allowing NET release to the extracellular milieu [22]. NET structure is composed of smooth strands of $15-17 \mathrm{~nm}$ diameter decorated with globular domains ranging from 25 to $50 \mathrm{~nm}[1,15]$.

Netosis is a new type of cell death, different from necrosis and apoptosis [22]. Under netosis, there is neither DNA fragmentation nor phosphatidylserine exposure in the outer membrane leaflet, hallmarks of these latter forms of cell death, respectively. The intact nuclear envelope differentiates necrosis from netosis, and NET-DNA was not detected in the culture supernatants of apoptotic or necrotic neutrophils [22]. Moreover, netosis seems to be independent of caspases and RIP-1 kinases, since pretreatment of neutrophils with zVAD-fmk or necrostatin-1 did not affect netosis completion $[15,56]$. All these features differentiate netosis from apoptosis and necrosis. However, it was recently shown that netosis induced by PMA was dependent on a simultaneous activation of both autophagy and superoxide production, and neither mechanism isolated was capable to induce netosis [56]. Thus, inhibition of autophagy prevents chromatin decondensation and netosis, without affecting superoxide production, demonstrating that NADPH oxidase activity is necessary, but insufficient to, alone, trigger netosis. Furthermore, induction of autophagy in neutrophils from chronic granulomatous disease patients, which possess a defective NADPH oxidase, thus, unable to generate reactive oxygen species (ROS), was not sufficient to promote chromatin decondensation [56].

In addition, netosis seems to occur by a mechanism independent of neutrophil granule exocytosis, since Rab27adeficient neutrophils, which are deficient in exocytosis, are able to release NETs [20].

Presently, the great majority of stimuli described to induce netosis are dependent on ROS production by the multienzyme complex NADPH oxidase. Drugs that inhibit NADPH enzyme, hence, inhibit NET release [22, 44, 57]. Moreover, neutrophils from patients with chronic granulomatous disease are incapable of forming NETs $[22,58,59]$.

However, a novel netosis process was recently described for the bacterium Staphylococcus aureus, occurring independently of ROS and neutrophil lysis [45]. In this new mechanism of netosis, that occurs very rapidly, vesicles containing DNA sprout from the nuclear membrane are extruded intact to the extracellular environment, where they break and release their chromatin content that traps and kills bacteria. This more rapid mechanism would precede the ROS-dependent NET release [45]. Similarly, ROS generation is not sufficient to rescue netosis from patients suffering from syndrome of neonatal neutrophil dysfunction, a disease associated with sepsis and other severe infections [57]. Another ROS-independent NET release was reported for Leishmania donovani [37].

It has been demonstrated at the molecular level that chromatin decondensation, a pivotal event during netosis, occurs with elastase migration from the primary granules to the neutrophil nuclei, where this enzyme partially degrades histones [33]. As follow, myeloperoxidase also migrate to the nucleus, synergizing with elastase for the chromatin decondensation by a still unknown mechanism, which is independent of its enzymatic activity. Importantly, neutrophils from myeloperoxidase-deficient patients are unable to release NET [60]. Treatment of neutrophils with specific neutrophil-elastase inhibitors abrogates NET formation, and purified neutrophil elastase was able to digest histones 


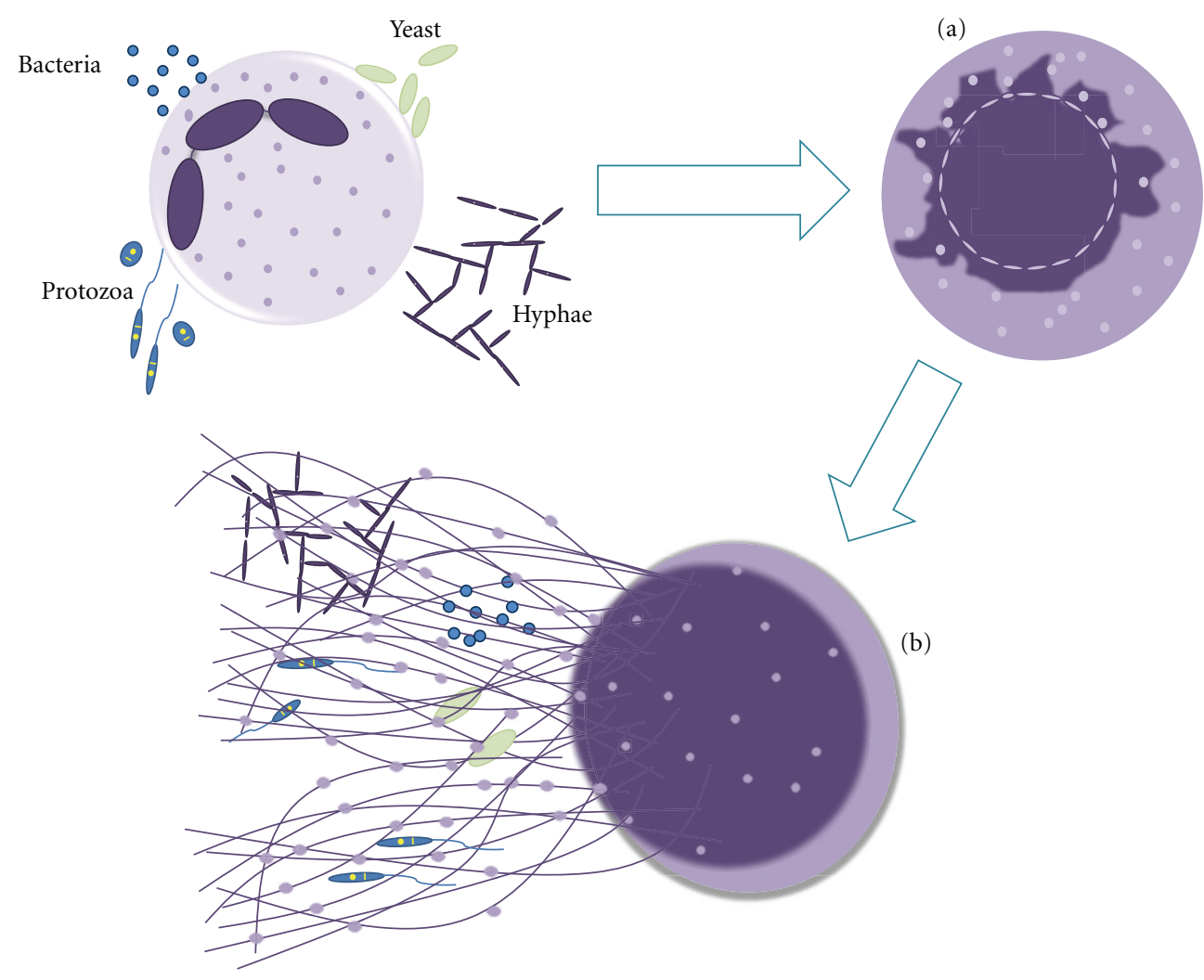

Figure 1: Mechanism of Neutrophil Extracellular Traps release. Neutrophils are stimulated by contact with bacteria, protozoan, fungi (yeast and hyphae forms) or their products (not shown), leading to: (a) ultrastructural alterations of nuclear shape with chromatin decondensation, swollen and fragmentation of the nuclear membrane, which allow the association of granules and cytoplasmic proteins with the chromatin, and (b) release of extracellular structures consisting of a DNA-backbone, decorated with histones, neutrophil granular and cytoplasmatic proteins (NETs), which ensnare and kill microorganisms.

and promote chromatin decondensation in isolated nuclei. Moreover, mice knockout for the elastase-encoding gene did not produce NET in a model of pulmonary infection by Klebsiella pneumoniae [33].

Another important event involved in chromatin decondensation is histone hypercitrullination, a reaction catalyzed by peptidyl arginine deiminase 4 (PAD4), in which histones' arginines are converted to citrullines by deimination. Hypercitrullinated histones were detected in NETs, but not in apoptotic neutrophils $[11,61]$. It has also been demonstrated that mice knockout for PAD4 enzyme are unable to form NETs upon activation by different stimuli, being deficient in bacterial killing by these traps [11, 62, 63]. Infection of $\mathrm{PAD}^{+/+}$and $\mathrm{PAD}^{-/-}$mice with group A Streptococcus confirmed that $\mathrm{PAD}^{-/-}$animals were more susceptible to infection, presenting more lesions than $\mathrm{PAD}^{+/+}$. Moreover, neutrophils purified from $\mathrm{PAD}^{-/-}$animals did not release NETs when stimulated by lipopolysaccharide or oxygen peroxide as their wild-type counterparts [62].

Hitherto, we know that upon protein kinase $\mathrm{C}$ activation by PMA or by diacylglycerol (DAG) analogs, RafMEK-ERK pathway is required for NET formation and also that this signaling pathway is upstream of NADPH oxidase activation, since diphenylene iodonium (DPI) did not abolish phosphorilation of ERK [27]. Moreover, the antiapoptotic protein myeloid cell leukemia ( $\mathrm{Mcl})-1$ is overexpressed in PMA-activated neutrophils, a pathway required for PMA/DAG/Helicobacter pylori NET induction [27].

The participation of Rac2, an isoform of Rac small GTPases, in NET induction by PMA or LPS-stimulated mice neutrophils has also been evidenced [44]. Rac2 null mice have negligible NET production in comparison to Rac1 null and wild-type counterparts, showing that Rac2 isoform is required for NET release. Rac2 mutants are unable of producing NETs due to a lack of ROS production, which is rescued by the addition of hydrogen peroxide to neutrophils. Since Rac has been shown to regulate nitric oxidase synthase (NOS), the role of nitric oxide (NO) on NET production by wild-type and Rac2 mutant mice was investigated. Indeed, L-NAME, an NOS inhibitor, reduced NET release induced by PMA in mice neutrophils [44]. Confirming the role of nitric oxide on NET production, 7-NI, another NOS inhibitor, also decreased NET release induced by PMA in human macrophages [43]. Intriguingly, treatment with SNAP, an NO donor, did not induce NET release either in wild-type or Rac2 null neutrophils. However, increased NET formation was observed by SNAP treatment of PMA-activated wild-type neutrophils, suggesting that ROS production by NADPH oxidase may be required for NET induction by NO [44]. These discrepancies could be due 
to the source of the neutrophils used in each study: SNAP induced netosis in human blood neutrophils [43] but not in mice bone marrow neutrophils [44].

The participation of NO in NET induction was also demonstrated using SNAP and SNP, two NO donors [43]. Human neutrophils treated with these compounds release NET in a dose- and time-dependent manner, which was inhibited by $\mathrm{N}$-acetyl cysteine, an ROS scavenger. SNAPinduced NET was abolished by DPI, an NADPH oxidase inhibitor, as well as by 4 -aminobenzoic acid hydrazide $(\mathrm{ABAH})$, a myeloperoxidase inhibitor, suggesting that NET induction by $\mathrm{NO}$ occurs with increasing free radicals generation [43].

\section{Functions of Neutrophil Extracellular Traps}

NETs grab microbes avoiding their dissemination through the organism, and also offering a high local concentration of antimicrobial proteins. Bactericidal activity of NETassociated histones has been proved against Shigella flexneri, Salmonella typhimurium, Salmonella enterica, Staphylococcus aureus, Streptococcus pyogenes, and Bacillus anthracis [1, 64]; however, it remains to be determined whether the bactericidal activity of histones is modulated by its hypercitrullination. In addition, the cytosolic calprotectin protein complex (S100A8/A9) associated to NETs kills Candida albicans and Cryptococcus neoformans [15].

Net-associated histones and calprotectin have been considered as bactericidal and fungicide, respectively, but many other NET-linked components are also endowed with microbicidal properties, at least in their free forms $[1,65,66]$. These molecules include cathelicidin LL-37, defensins, bacterial permeability-increasing protein (BPI), lactoferrin, myeloperoxidase, proteinase 3 , and elastase $[3,4$, 67]. Furthermore, these components could act synergistically as shown for two of them, the peptidoglycan recognition protein-S and lysozyme, which colocalize in NETs [67].

Even though resistant to NET-mediated killing, group A Streptococcus, S. pneumoniae, Mycobacterium tuberculosis, and Haemophilus influenza are caught by NETs, suggesting that this property could also have an important role for the host immune response $[25,26,42]$.

Presently, it is unknown how so diverse and different microorganisms are ensnared by NETs. Many of the NETs constituents are highly cationic, and it is likely that NETs can bind negatively charged surfaces, while NET-specific recognition sites for microorganisms could not be excluded [68].

Albeit NETs are toxic for microorganisms, some microbes are able to escape the NET-mediated killing. To name a few of these strategies, the capsule expression and M1 protein of group A Streptococcus are important to NET resistance [69]. In addition, endonucleases expressed by Staphylococcus, Streptococcus pneumonia, and group A Streptococcus enhance bacterium survival by digesting NET-DNA scaffold [34, 49, 70-72]. Importantly, since DNA constitutes the NET backbone, its digestion with DNase rescues microorganisms from NET-mediated killing $[1,13,35]$. NET-escape mechanisms as well as
NET role on autoimmune diseases are reviewed elsewhere $[13,73-77]$.

\section{Cells Able to Release Extracellular Traps (ETosis)}

Netosis was first described in neutrophils, thus the origin of its name. However, other cells such as eosinophils and mast cells also release extracellular traps (ETs) composed by DNA and antimicrobial proteins $[28,30]$. Monocytes and macrophages were also shown to release extracellular traps, but to a lesser extent when compared to neutrophils, and the microbicidal activity of these ETs has not yet been determined [21, 78]. Since it seems to be a more general mechanism, shared by different cell types, the release of intracellular DNA to the extracellular milieu was renamed as ETosis, meaning death with release of DNA extracellular traps. Interestingly, eosinophils release their mitochondrial DNA, in a death-independent way [30]. These same authors reported that GM-CSF-primed neutrophils released mitochondrial DNA associated with granule proteins, forming neutrophil extracellular traps in response to LPS or complement C5a fragment [24].

Besides occurring in different cell types, ETosis seems to be a well-conserved mechanism, since release of ETs was reported for neutrophils or related cells in many different organisms, such as ox, horse, fish, mouse, and cat neutrophils, as well as chicken heterophils $[12,17-19,29$, 36, 38, 39, 44]. Although classical ETs were not observed in Galleria mellonella, DNA derived from oenocytoid cells participates in the haemolymph coagulation, an important mechanism for microbes killing in insects [79].

Interestingly, ETs were also evidenced in plants and seem to play an important role on root tip defense against fungal infections, implying a similar behavior between plant root and animal cells, which extrude ETs in a defense mechanism important for plant growth and survival $[80,81]$.

Because it was first described in neutrophils, which are easy cells to work with, the majority of the data available analyze netosis aspects, mainly when the studies were related to infectious diseases, although it has an important role in autoimmune diseases such as systemic lupus erythematosus and vasculitis [75-77].

\section{NETs and Protozoa}

Neutrophils are rapidly recruited to infection sites during infections with many protozoa and microbial pathogens, pointing out the importance of these phagocytes in the immune response to these infectious agents. Leishmania particularly meets neutrophils in the very beginning of the infection process, since these protozoan parasites are inoculated by the sand fly vector in a pool of blood. Thus, based on the interaction between Leishmania and neutrophils, we investigate the capacity of this trypanosomatid to induce netosis, to better understand the first steps of the Leishmania infection [35]. Initially, we detected that both forms of the parasite, promastigotes and amastigotes, 
were able to induce NET extrusion in human neutrophils and were caught by the resulting meshes (Figure 2; see Supplementary video in supplementary material available online at doi:10.1155/2012/929743). In addition, we demonstrated that the glycoconjugate lipophosphoglycan, which is expressed on the promastigote cell surface, induced neutrophils to release NET in a dose-dependent manner. NETs were toxic for the promastigotes, an effect mediated by the histones present in the lattices, whose toxicity to the parasite was neutralized by antihistone antibodies [35]. The ability of histones to kill Leishmania was further confirmed by parasite death upon its exposure to histones purified from calf thymus (Figure 3). NETs occurrence goes further beyond experimental findings since the presence of NETs in lesion biopsies of patients with active cutaneous leishmaniasis was also shown [35].

Leishmania sensitivity to histone-mediated toxicity also includes promastigotes of $L$. mexicana, $L$. braziliensis, $L$. major, and L. amazonensis, although the death mechanism mediated by these proteins was still unknown [82]. Interestingly, amastigotes from $L$. mexicana and L. amazonensis were resistant to the histone-mediated toxicity [82]. NET induction was also shown for L. donovani promastigotes, but, contrary to the former findings, the authors reported that both $L$. donovani and $L$. major promastigotes were resistant to the NET-mediated killing [37].

Apicomplexans such as Plasmodium, the causative agent of malaria, and Eimeria, the causative agent of eimeriosis in cattle, comprise some of the most life-threatening protozoan parasites. Although a direct NET induction by Plasmodium has not been described yet, there is one report showing NETs in the blood of Plasmodium falciparum-infected children, showing infected erythrocytes and trophozoites attached to structures identified as NET by DNA staining [83].

Behrendt and coworkers [16] reported that sporozoites of Eimeria bovis induce NET extrusion by calf neutrophils, and this traps snare sporozoites. Although a NET-lethal effect on Eimeria sporozoites was not demonstrated, the parasite infectivity to bovine primary endothelial cells was decreased in comparison to untreated parasites. Thus, arrest of Eimeria sporozoites by NETs may prevent host cell invasion required for this parasite replication.

\section{NETs and Fungi}

Fungal pathogens cause a wide and increasing number of severe infections with high mortality rates, mainly in immunocompromised individuals. The phenomenon of NET release was observed in experimental models of fungal infections using Candida albicans, Cryptococcus neoformans, Aspergillus nidulans, A. fumigatus, and Cryptococcus gattii $[8,13,15,58,84]$.

C. albicans is the major cause of mycoses in humans, which can range from mild superficial infection of the skin to severe disseminated systemic disease. In vitro studies have shown that hyphae and yeast forms of $C$. albicans induce and are trapped and killed by NETs released from human neutrophils, as well as $C$. neoformans $[13,15]$. Calprotectins,

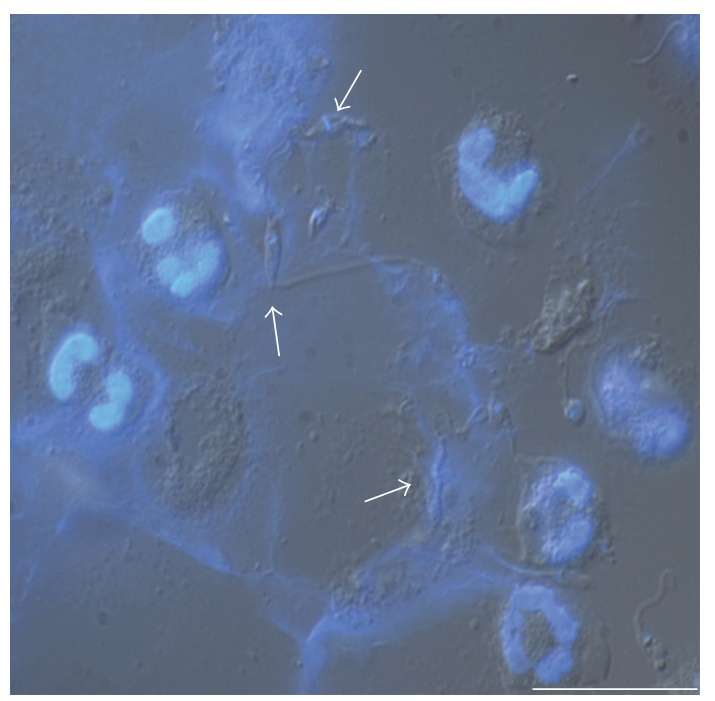

FIGURE 2: Immunostaining of NETs induced by Leishmania. Naïve neutrophils were incubated with promastigotes $(1: 5$ ratio) for $1 \mathrm{~h}$ at $35^{\circ} \mathrm{C}$. Cells were fixed and stained with DAPI and shown merged with differential interference contrast image. Arrows point to NETensnared promastigotes (Bars: $20 \mu \mathrm{m}$ ).

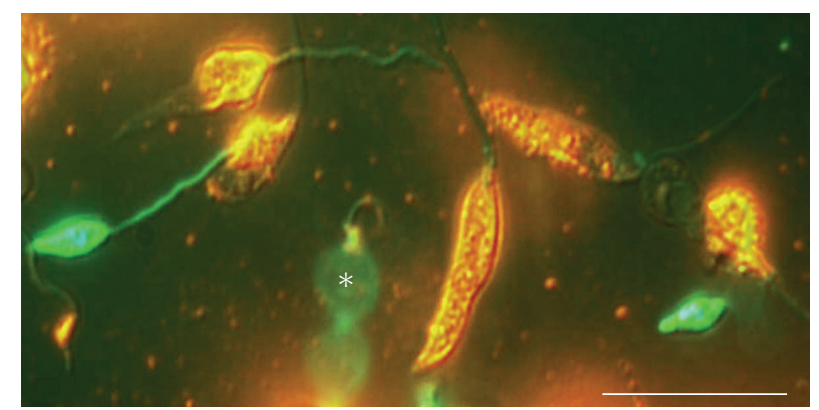

Figure 3: Histone toxicity to promastigotes. Promastigotes were incubated with purified histone for $30 \mathrm{~min}$ and stained by the live/dead method. Dead promastigotes stained in yellow/orange and live promastigotes in green. Differential interference contrast image merged with the fluorescence staining of the same cells. (*) The beating of a live promastigote flagellum. Bars, $20 \mu \mathrm{m}$.

a calcium-binding cytoplasmic heterodimeric protein complex, were shown as the major antifungal component associated to the NETs, in vitro and in vivo. Calprotectin chelates essential metal ions such as $\mathrm{Zn}^{2+}$ and $\mathrm{Mn}^{2+}$ resulting in reduced $C$. albicans growth in subcutaneous and pulmonary infection of mice $[15,85]$. Moreover, immunodepletion of calprotectin abolished the growth inhibitory activity of NETs in vitro, and calprotectin-deficient mice were unable to clear C. albicans infection [15].

The role of calprotectin against Aspergillus was also established in calprotectin knockout mice, which lost the ability to control the fungal infection [86]. On the contrary, an in vitro study using A. fumigatus showed that although resting conidia and germinal tube forms are able to induce NETs, the webs did not kill the fungus. The control of swollen conidia germination is achieved by phagocytosis, 
although NET inhibits polar growth of germ tube forms in a calprotectin-dependent manner [9].

In a human case of an invasive pulmonary aspergillosis caused by $A$. nidulans and refractory to therapy, the role of NET formation was pointed out through a successful restoration of the immune response against this fungus by gene therapy, in an 8.5-years-old boy with chronic granulomatous disease. Restoring NADPH oxidase expression after gp91 phox gene transfer, neutrophils defense against the conidia and hyphae forms of $A$. nidulans was reestablished in the treated patient [58]. This study unequivocally proved the role of ROS production for NET induction, at least for fungi.

Another study showed that, in a mice model of $A$. fumigatus-lung infection, NETs arise in 3-4 hours after immigrating neutrophils reach the infectious focus. Moreover, high amount of NETs against hyphae forms were observed in comparison to conidia. The presence of hydrophobin RodA, the major component of resting conidia surface, reduced NET formation. This fungal protein was identified as being an important factor to protect conidia from recognition [87], and now it seems to be an important factor to prevent triggering of NET extrusion; however, the molecular mechanism behind prevention of NET formation remains to be elucidated [88].

The encapsulated yeast C. gatti, is the agent of one-tenth to one-third of pulmonary cryptococcosis and meningitis worldwise. This species is distinguished from C. neoformans by its occurrence in trees, rather than pigeon droppings. Special diagnostic tools used to differentiate these species include the cigar-shaped yeast morphology in the host cerebrospinal fluid, agglutinating serotype, creatine assimilation, and elongated rod-shaped basidiospores [14, 84]. A study analyzing neutrophils, interaction with C. gatti showed an extensively NET formation in vitro, but without correlation with fungal killing. Moreover, comparison studies demonstrate that $C$. gatti, expressing extracellular fibrils were more resistant to neutrophil killing than capsulate mutants [14].

Whether NETs really have a role in the control of fungal infections needs to be better explored, thence it is not the only mechanism, although it means an important tool to detain infection.

\section{NETs and Viruses}

Although presently there are no data about virus capacity to directly induce NET release, the role of netosis in viral infections has been addressed for feline leukemia virus $(\mathrm{FeLV})$ and influenza A virus infections $[38,63,89]$.

Our group described that netosis of cat neutrophils could be modulated by the feline leukemia virus (FeLV) infection [36]. In fact, neutrophils from FeLV (-) and from asymptomatic FeLV $(+)$ cats release NETs when stimulated with PMA or Leishmania. However, neutrophils from FeLV (+) symptomatic cats spontaneously release high quantities of NETs in comparison to either the neutrophils from FeLV $(-)$ or from asymptomatic FeLV $(+)$ cats. On the other hand, neutrophils from FeLV (+) symptomatic cats do not respond to NET-releasing stimuli. Our data suggest that netosis could be related to disease status, at least in FeLV-feline infection, and that this feature could be used as a diagnostic tool [36].

In a recent study, NET was induced in a murine model of influenza A virus (A/Puerto Rico/8/34 H1N1) pneumonitis and correlated to lesions in the alveoli and bronchioles, leading to complications of acute respiratory distress syndrome [89]. Also analyzing infection with influenza A virus (A/WSN/33/H1N1 strain) in NET-impaired mice (PAD4 knocked out) and in wild-type counterparts, NET induction was found in the bronchoalveolar lavage (BAL) of the wildtype mice, but not in the PAD4 ${ }^{-1-}$ mice [63]. Interestingly, neutrophils obtained from lungs of wild-type mice release NET upon contact with influenza A-infected alveolar epithelial cells. However, no differences in morbidity and mortality were observed in both mice strains, ruling out the hypothesis that NET could mediate viral clearance in this model of acute infection [63].

\section{In Vivo Detection of ETosis}

The physiologic role of ETs can be supported by its abundance in sites of infected and noninfected models of inflammation. Accordingly, etosis has been reported in spontaneous human appendicitis [1], experimental dysentery induced by Shigella in rabbits [1], infections by Streptococcus [90], pneumonia by Streptococcus pneumonia [90], blood of children infected by Plasmodium falciparum [83], periodontitis [91], infections by Aspergillus nidulans [58] and by Eimeria bovis [16], as well as in pulmonary Aspergillus fumigatus infections [8], and cutaneous lesions of leishmaniasis [35]. This phenomenon was also demonstrated to be relevant in human preeclampsia [31], in small vessels vasculitis [50], in systemic lupus erythematosus [7, 50, 77], on thrombosis [92], and in psoriasis [32].

\section{Closing Remarks}

Although etosis was recently described [1], the literature concerning this new form of cell-mediated killing is growing quickly. Even though many advances have been achieved recently, the molecular mechanism underlying NET release is far from being understood. Different works have pointed out that only around $30 \%$ of the neutrophils stimulated by different stimuli dye by netosis. Many different microorganisms and molecules induce NET release, but it is unknown if netosis is triggered by common or different signaling pathways. Besides, many of the stimuli that induce NET release were formerly also described as inducers of other neutrophil functions, such as phagocytosis or chemotaxis. Thus, it is important to determine how and why some neutrophils, facing a parasite for instance, activate the netosis process, while others in the same population phagocytose the parasite. Neutrophils were short-lived cells and, presently, no markers of subpopulations are available to test if the different outcomes reflect distinct neutrophil subpopulations. Also, the maturing status of these cells could contribute for these differences, or the cytokine milieu as has been reported for polarized tumor-associated neutrophils [93]. 
Anyhow, netosis, or etosis in general, must be a strictly regulated process since it is a death mechanism, which released components, could participate either in antimicrobial defense and/or harm host tissues, and induce autoimmunity, where these structures are gaining increased relevance.

ETosis is proving to be a critical mechanism of host defense, offering new potential for disease control and defining new targets for intervening on infectious and also autoimmune diseases, besides grant novel tools for diagnosis [94] and/or prognosis [95].

\section{Acknowledgments}

The authors thank Dr. Dumith Chequer Bou-Habib (Lab de Pesquisa sobre o Timo, Instituto Oswaldo Cruz, Rio de Janeiro, Brazil) for critically reading the paper and for his helpful comments and Dr. Flávio Lara (Lab de Microbiologia Celular, Instituto Oswaldo Cruz, Rio de Janeiro, Brazil) for the video microscopy analysis. They are also grateful to the Hemotherapy Service Hospital Clementino Fraga Filho (Universidade Federal do Rio de Janeiro, Rio de Janeiro, Brazil) for buffy coats. This work was supported by Conselho Nacional de Desenvolvimento Científico e Tecnológico (CNPq), Coordenação de Aperfeiçoamento de Pessoal de Nível Superior (CAPES), and Fundação Carlos Chagas Filho de Amparo à Pesquisa do Estado do Rio de Janeiro (FAPERJ).

\section{References}

[1] V. Brinkmann, U. Reichard, C. Goosmann et al., "Neutrophil extracellular traps kill bacteria," Science, vol. 303, no. 5663, pp. 1532-1535, 2004.

[2] V. Brinkmann and A. Zychlinsky, "Beneficial suicide: why neutrophils die to make NETs," Nature Reviews Microbiology, vol. 5, no. 8, pp. 577-582, 2007.

[3] V. Kumar and A. Sharma, "Neutrophils: cinderella of innate immune system," International Immunopharmacology, vol. 10, no. 11, pp. 1325-1334, 2010.

[4] N. Borregaard, "Neutrophils, from marrow to microbes," Immunity, vol. 33, no. 5, pp. 657-670, 2010.

[5] A. Mantovani, M. A. Cassatella, C. Costantini, and S. Jaillon, "Neutrophils in the activation and regulation of innate and adaptive immunity," Nature Reviews Immunology, vol. 11, no. 8, pp. 519-531, 2011.

[6] A. K. Gupta, M. B. Joshi, M. Philippova et al., "Activated endothelial cells induce neutrophil extracellular traps and are susceptible to NETosis-mediated cell death," FEBS Letters, vol. 584, no. 14, pp. 3193-3197, 2010.

[7] G. S. Garcia-Romo, S. Caielli, B. Vega et al., "Netting neutrophils are major inducers of type I IFN production in pediatric systemic lupus erythematosus," Science Translational Medicine, vol. 3, no. 73, p. 73ra20, 2011.

[8] S. Bruns, O. Kniemeyer, M. Hasenberg et al., "Production of extracellular traps against Aspergillus fumigatus in vitro and in infected lung tissue is dependent on invading neutrophils and influenced by hydrophobin RodA," PLoS pathogens, vol. 6, no. 4, Article ID e1000873, 2010.

[9] A. McCormick, L. Heesemann, J. Wagener et al., "NETs formed by human neutrophils inhibit growth of the pathogenic mold Aspergillus fumigatus," Microbes and Infection, vol. 12, no. 12-13, pp. 928-936, 2010.
[10] R. Lande, D. Ganguly, V. Facchinetti et al., "Neutrophils activate plasmacytoid dendritic cells by releasing self-DNApeptide complexes in systemic lupus erythematosus," Science Translational Medicine, vol. 3, no. 73, p. 73ra19, 2011.

[11] Y. Wang, M. Li, S. Stadler et al., "Histone hypercitrullination mediates chromatin decondensation and neutrophil extracellular trap formation," Journal of Cell Biology, vol. 184, no. 2, pp. 205-213, 2009.

[12] D. Palić, J. Ostojić, C. B. Andreasen, and J. A. Roth, "Fish cast NETs: neutrophil extracellular traps are released from fish neutrophils," Developmental and Comparative Immunology, vol. 31, no. 8, pp. 805-816, 2007.

[13] C. F. Urban, U. Reichard, V. Brinkmann, and A. Zychlinsky, "Neutrophil extracellular traps capture and kill Candida albicans and hyphal forms," Cellular Microbiology, vol. 8, no. 4, pp. 668-676, 2006.

[14] D. J. Springer, P. Ren, R. Raina et al., "Extracellular fibrils of pathogenic yeast Cryptococcus gattii are important for ecological niche, murine virulence and human neutrophil interactions," PLoS ONE, vol. 5, no. 6, Article ID e10978, 2010.

[15] C. F. Urban, D. Ermert, M. Schmid et al., "Neutrophil extracellular traps contain calprotectin, a cytosolic protein complex involved in host defense against Candida albicans," PLoS Pathogens, vol. 5, no. 10, Article ID e1000639, 2009.

[16] J. H. Behrendt, A. Ruiz, H. Zahner, A. Taubert, and C. Hermosilla, "Neutrophil extracellular trap formation as innate immune reactions against the apicomplexan parasite Eimeria bovis," Veterinary Immunology and Immunopathology, vol. 133, no. 1, pp. 1-8, 2010.

[17] J. D. Lippolis, T. A. Reinhardt, J. P. Goff, and R. L. Horst, "Neutrophil extracellular trap formation by bovine neutrophils is not inhibited by milk," Veterinary Immunology and Immunopathology, vol. 113, no. 1-2, pp. 248-255, 2006.

[18] A. S. Alghamdi and D. N. Foster, "Seminal DNase frees spermatozoa entangled in neutrophil extracellular traps," Biology of Reproduction, vol. 73, no. 6, pp. 1174-1181, 2005.

[19] N. Grinberg, S. Elazar, I. Rosenshine, and N. Y. Shpigel, “ $\beta$ hydroxybutyrate abrogates formation of bovine neutrophil extracellular traps and bactericidal activity against mammary pathogenic Escherichia coli," Infection and Immunity, vol. 76, no. 6, pp. 2802-2807, 2008.

[20] D. B. Munafo, J. L. Johnson, A. A. Brzezinska, B. A. Ellis, M. R. Wood, and S. D. Catz, "DNase i inhibits a late phase of reactive oxygen species production in neutrophils," Journal of Innate Immunity, vol. 1, no. 6, pp. 527-542, 2009.

[21] S. J. Webster, M. Daigneault, M. A. Bewley et al., "Distinct cell death programs in monocytes regulate innate responses following challenge with common causes of invasive bacterial disease," Journal of Immunology, vol. 185, no. 5, pp. 29682979, 2010.

[22] T. A. Fuchs, U. Abed, C. Goosmann et al., "Novel cell death program leads to neutrophil extracellular traps," Journal of Cell Biology, vol. 176, no. 2, pp. 231-241, 2007.

[23] S. Martinelli, M. Urosevic, A. Baryadel et al., "Induction of genes mediating interferon-dependent extracellular trap formation during neutrophil differentiation," Journal of Biological Chemistry, vol. 279, no. 42, pp. 44123-44132, 2004.

[24] S. Yousefi, C. Mihalache, E. Kozlowski, I. Schmid, and H. U. Simon, "Viable neutrophils release mitochondrial DNA to form neutrophil extracellular traps," Cell Death and Differentiation, vol. 16, no. 11, pp. 1438-1444, 2009.

[25] W. Hong, R. A. Juneau, B. Pang, and W. E. Swords, "Survival of bacterial biofilms within neutrophil extracellular traps promotes nontypeable haemophilus influenzae persistence 
in the chinchilla model for otitis media," Journal of Innate Immunity, vol. 1, no. 3, pp. 215-224, 2009.

[26] R. A. Juneau, B. Pang, K. E. D. Weimer, C. E. Armbruster, and W. E. Swords, "Nontypeable haemophilus influenzae initiates formation of neutrophil extracellular traps," Infection and Immunity, vol. 79, no. 1, pp. 431-438, 2011.

[27] A. Hakkim, T. A. Fuchs, N. E. Martinez et al., "Activation of the Raf-MEK-ERK pathway is required for neutrophil extracellular trap formation," Nature Chemical Biology, vol. 7, no. 2, pp. 75-77, 2011.

[28] M. von Köckritz-Blickwede, O. Goldmann, P. Thulin et al., "Phagocytosis-independent antimicrobial activity of mast cells by means of extracellular trap formation," Blood, vol. 111, no. 6, pp. 3070-3080, 2008.

[29] P. Chuammitri, J. Ostojić, C. B. Andreasen, S. B. Redmond, S. J. Lamont, and D. Palić, "Chicken heterophil extracellular traps (HETs): novel defense mechanism of chicken heterophils," Veterinary Immunology and Immunopathology, vol. 129, no. 1-2, pp. 126-131, 2009.

[30] S. Yousefi, J. A. Gold, N. Andina et al., "Catapult-like release of mitochondrial DNA by eosinophils contributes to antibacterial defense," Nature Medicine, vol. 14, no. 9, pp. 949 953, 2008.

[31] A. K. Gupta, P. Hasler, W. Holzgreve, S. Gebhardt, and S. Hahn, "Induction of neutrophil extracellular DNA lattices by placental microparticles and IL- 8 and their presence in preeclampsia," Human Immunology, vol. 66, no. 11, pp. 11461154, 2005.

[32] A. M. Lin, C. J. Rubin, R. Khandpur et al., "Mast cells and neutrophils release IL-17 through extracellular trap formation in psoriasis," Journal of Immunology, vol. 187, no. 1, pp. 490500, 2011.

[33] V. Papayannopoulos, K. D. Metzler, A. Hakkim, and A. Zychlinsky, "Neutrophil elastase and myeloperoxidase regulate the formation of neutrophil extracellular traps," Journal of Cell Biology, vol. 191, no. 3, pp. 677-691, 2010.

[34] J. T. Buchanan, A. J. Simpson, R. K. Aziz et al., "DNase expression allows the pathogen group A Streptococcus to escape killing in neutrophil extracellular traps," Current Biology, vol. 16, no. 4, pp. 396-400, 2006.

[35] A. B. Guimarães-Costa, M. T. C. Nascimento, G. S. Froment et al., "Leishmania amazonensis promastigotes induce and are killed by neutrophil extracellular traps," Proceedings of the National Academy of Sciences of the United States of America, vol. 106, no. 16, pp. 6748-6753, 2009.

[36] A. B. Wardini, A. B. Guimarães-Costa, M. T. C. Nascimento et al., "Characterization of neutrophil extracellular traps in cats naturally infected with feline leukemia virus," Journal of General Virology, vol. 91, no. 1, pp. 259-264, 2010.

[37] C. Gabriel, W. R. McMaster, D. Girard, and A. Descoteaux, "Leishmania donovani promastigotes evade the antimicrobial activity of neutrophil extracellular traps," Journal of Immunology, vol. 185, no. 7, pp. 4319-4327, 2010.

[38] D. Ermert, C. F. Urban, B. Laube, C. Goosmann, A. Zychlinsky, and V. Brinkmann, "Mouse neutrophil extracellular traps in microbial infections," Journal of Innate Immunity, vol. 1, no. 3, pp. 181-193, 2009.

[39] N. A. Aulik, K. M. Hellenbrand, H. Klos, and C. J. Czuprynski, "Mannheimia haemolytica and its leukotoxin cause neutrophil extracellular trap formation by bovine neutrophils," Infection and Immunity, vol. 78, no. 11, pp. 4454-4466, 2010.

[40] X. Lauth, M. von Köckritz-Blickwede, C. W. McNamara et al., "M1 protein allows group a streptococcal survival in phagocyte extracellular traps through cathelicidin inhibition," Journal of Innate Immunity, vol. 1, no. 3, pp. 202-214, 2009.

[41] S. Oehmcke, M. Mörgelin, and H. Herwald, "Activation of the human contact system on neutrophil extracellular traps," Journal of Innate Immunity, vol. 1, no. 3, pp. 225-230, 2009.

[42] V. Ramos-Kichik, R. Mondragón-Flores, M. MondragónCastelán et al., "Neutrophil extracellular traps are induced by Mycobacterium tuberculosis," Tuberculosis, vol. 89, no. 1, pp. 29-37, 2009.

[43] S. Patel, S. Kumar, A. Jyoti et al., "Nitric oxide donors release extracellular traps from human neutrophils by augmenting free radical generation," Nitric Oxide, vol. 22, no. 3, pp. 226234, 2010.

[44] M. B. H. Lim, J. W. P. Kuiper, A. Katchky, H. Goldberg, and M. Glogauer, "Rac2 is required for the formation of neutrophil extracellular traps," Journal of Leukocyte Biology, vol. 90, no. 4, pp. 771-776, 2011.

[45] F. H. Pilsczek, D. Salina, K. K. H. Poon et al., "A novel mechanism of rapid nuclear neutrophil extracellular trap formation in response to Staphylococcus aureus," Journal of Immunology, vol. 185, no. 12, pp. 7413-7425, 2010.

[46] S. R. Clark, A. C. Ma, S. A. Tavener et al., "Platelet TLR4 activates neutrophil extracellular traps to ensnare bacteria in septic blood," Nature Medicine, vol. 13, no. 4, pp. 463-469, 2007.

[47] O. A. Chow, M. von Köckritz-Blickwede, A. T. Bright et al., "Statins enhance formation of phagocyte extracellular traps," Cell Host and Microbe, vol. 8, no. 5, pp. 445-454, 2010.

[48] L. E. Crotty Alexander, H. C. Maisey, A. M. Timmer et al., "M1T1 group A streptococcal pili promote epithelial colonization but diminish systemic virulence through neutrophil extracellular entrapment," Journal of Molecular Medicine, vol. 88, no. 4, pp. 371-381, 2010.

[49] K. Beiter, F. Wartha, B. Albiger, S. Normark, A. Zychlinsky, and B. Henriques-Normark, "An endonuclease allows Streptococcus pneumoniae to escape from neutrophil extracellular traps," Current Biology, vol. 16, no. 4, pp. 401-407, 2006.

[50] K. Kessenbrock, M. Krumbholz, U. Schönermarck et al., "Netting neutrophils in autoimmune small-vessel vasculitis," Nature Medicine, vol. 15, no. 6, pp. 623-625, 2009.

[51] D. Palić, C. B. Andreasen, J. Ostojić, R. M. Tell, and J. A. Roth, "Zebrafish (Danio rerio) whole kidney assays to measure neutrophil extracellular trap release and degranulation of primary granules," Journal of Immunological Methods, vol. 319, no. 1-2, pp. 87-97, 2007.

[52] S. Casutt-Meyer, F. Renzi, M. Schmaler, N. J. Jann, M. Amstutz, and G. R. Cornelis, "Oligomeric coiled-coil adhesin YadA is a double-edged sword," PLoS ONE, vol. 5, no. 12, Article ID e15159, pp. 151-159, 2010.

[53] A. L. Cogen, K. Yamasaki, J. Muto et al., "Staphylococcus epidermidis antimicrobial $\delta$-toxin (phenol-soluble modulin$\gamma$ ) cooperates with host antimicrobial peptides to kill group A Streptococcus," PLoS ONE, vol. 5, no. 1, Article ID e8557, 2010.

[54] H. C. Ju, I. P. Fraser, K. Fukase et al., "Human peptidoglycan recognition protein $S$ is an effector of neutrophil-mediated innate immunity," Blood, vol. 106, no. 7, pp. 2551-2558, 2005.

[55] S. Jaillon, G. Peri, Y. Delneste et al., "The humoral pattern recognition receptor PTX3 is stored in neutrophil granules and localizes in extracellular traps," Journal of Experimental Medicine, vol. 204, no. 4, pp. 793-804, 2007.

[56] Q. Remijsen, T. V. Berghe, E. Wirawan et al., "Neutrophil extracellular trap cell death requires both autophagy and 
superoxide generation," Cell Research, vol. 21, no. 2, pp. 290304, 2011.

[57] C. C. Yost, M. J. Cody, E. S. Harris et al., "Impaired neutrophil extracellular trap (NET) formation: a novel innate immune deficiency of human neonates," Blood, vol. 113, no. 25, pp. 6419-6427, 2009.

[58] M. Bianchi, A. Hakkim, V. Brinkmann et al., "Restoration of NET formation by gene therapy in CGD controls aspergillosis," Blood, vol. 114, no. 13, pp. 2619-2622, 2009.

[59] V. Brinkmann and A. Zychlinsky, "Beneficial suicide: why neutrophils die to make NETs," Nature Reviews Microbiology, vol. 5, no. 8, pp. 577-582, 2007.

[60] K. D. Metzler, T. A. Fuchs, W. M. Nauseef et al., "Myeloperoxidase is required for neutrophil extracellular trap formation: implications for innate immunity," Blood, vol. 117, no. 3, pp. 953-959, 2011.

[61] I. Neeli, S. N. Khan, and M. Radic, "Histone deimination as a response to inflammatory stimuli in neutrophils," Journal of Immunology, vol. 180, no. 3, pp. 1895-1902, 2008.

[62] P. Li, M. Li, M. R. Lindberg, M. J. Kennett, N. Xiong, and Y. Wang, "PAD4 is essential for antibacterial innate immunity mediated by neutrophil extracellular traps," Journal of Experimental Medicine, vol. 207, no. 9, pp. 1853-1862, 2010.

[63] S. Hemmers, J. R. Teijaro, S. Arandjelovic, and K. A. Mowen, "PAD4-mediated neutrophil extracellular trap formation is not required for immunity against influenza infection," PLoS ONE, vol. 6, no. 7, Article ID e22043, 2011.

[64] H. Kawasaki and S. Iwamuro, "Potential roles of histones in host defense as antimicrobial agents," Infectious DisordersDrug Targets, vol. 8, no. 3, pp. 195-205, 2008.

[65] R. Bucki, K. Leszczyńska, A. Namiot, and W. Sokołowski, "Cathelicidin LL-37: a multitask antimicrobial peptide," Archivum Immunologiae et Therapiae Experimentalis, vol. 58, no. 1, pp. 15-25, 2010.

[66] C. Nathan, "Neutrophils and immunity: challenges and opportunities," Nature Reviews Immunology, vol. 6, no. 3, pp. 173-182, 2006.

[67] H. C. Ju, I. P. Fraser, K. Fukase et al., "Human peptidoglycan recognition protein $\mathrm{S}$ is an effector of neutrophil-mediated innate immunity," Blood, vol. 106, no. 7, pp. 2551-2558, 2005.

[68] V. Brinkmann and A. Zychlinsky, "Beneficial suicide: why neutrophils die to make NETs," Nature Reviews Microbiology, vol. 5, no. 8, pp. 577-582, 2007.

[69] J. N. Cole, M. A. Pence, M. von Köckritz-Blickwede et al., " $\mathrm{M}$ protein and hyaluronic acid capsule are essential for in vivo selection of covRS mutations characteristic of invasive serotype M1T1 group A Streptococcus," mBio, vol. 1, no. 4, Article ID e00191-10, 2010.

[70] E. T. M. Berends, A. R. Horswill, N. M. Haste, M. Monestier, V. Nizet, and M. Von Köckritz-Blickwede, "Nuclease expression by Staphylococcus aureus facilitates escape from neutrophil extracellular traps," Journal of Innate Immunity, vol. 2, no. 6, pp. 576-586, 2010.

[71] P. Sumby, K. D. Barbian, D. J. Gardner et al., "Extracellular deoxyribonuclease made by group A Streptococcus assists pathogenesis by enhancing evasion of the innate immune response," Proceedings of the National Academy of Sciences of the United States of America, vol. 102, no. 5, pp. 1679-1684, 2005.

[72] M. J. Walker, A. Hollands, M. L. Sanderson-Smith et al., "DNase Sdal provides selection pressure for a switch to invasive group A streptococcal infection," Nature Medicine, vol. 13, no. 8, pp. 981-985, 2007.
[73] M. von Köckritz-Blickwede and V. Nizet, "Innate immunity turned inside-out: antimicrobial defense by phagocyte extracellular traps," Journal of Molecular Medicine, vol. 87, no. 8, pp. 775-783, 2009.

[74] C. F. Urban, S. Lourido, and A. Zychlinsky, "How do microbes evade neutrophil killing?” Cellular Microbiology, vol. 8, no. 11, pp. 1687-1696, 2006.

[75] P. Lamprecht, J. Holle, and W. L. Gross, "Update on clinical, pathophysiological and therapeutic aspects in ANCAassociated vasculitides," Current Drug Discovery Technologies, vol. 6, no. 4, pp. 241-251, 2009.

[76] J. C. Jennette, R. J. Falk, and A. H. Gasim, "Pathogenesis of antineutrophil cytoplasmic autoantibody vasculitis," Current Opinion in Nephrology and Hypertension, vol. 20, no. 3, pp. 263-270, 2011.

[77] A. Hakkim, B. G. Fürnrohr, K. Amann et al., "Impairment of neutrophil extracellular trap degradation is associated with lupus nephritis," Proceedings of the National Academy of Sciences of the United States of America, vol. 107, no. 21, pp. 9813-9818, 2010.

[78] M. Bartneck, H. A. Keul, Z. K. Gabriele, and J. Groll, "Phagocytosis independent extracellular nanoparticle clearance by human immune cells," Nano Letters, vol. 10, no. 1, pp. 59-64, 2010.

[79] B. Altincicek, S. Stötzel, M. Wygrecka, K. T. Preissner, and A. Vilcinskas, "Host-derived extracellular nucleic acids enhance innate immune responses, induce coagulation, and prolong survival upon infection in insects," Journal of Immunology, vol. 181, no. 4, pp. 2705-2712, 2008.

[80] F. Wen, G. J. White, H. D. Vanetten, Z. Xiong, and M. C. Hawes, "Extracellular DNA is required for root tip resistance to fungal infection," Plant Physiology, vol. 151, no. 2, pp. 820$829,2009$.

[81] M. C. Hawes, G. Curlango-Rivera, F. Wen, G. J. White, H. D. VanEtten, and Z. Xiong, "Extracellular DNA: the tip of root defenses?” Plant Science, vol. 180, no. 6, pp. 741-745, 2011.

[82] Y. Wang, Y. Chen, L. Xin et al., "Differential microbicidal effects of human histone proteins $\mathrm{H} 2 \mathrm{~A}$ and $\mathrm{H} 2 \mathrm{~B}$ on Leishmania promastigotes and amastigotes," Infection and Immunity, vol. 79, no. 3, pp. 1124-1133, 2011.

[83] V. S. Baker, G. E. Imade, N. B. Molta et al., "Cytokineassociated neutrophil extracellular traps and antinuclear antibodies in Plasmodium falciparum infected children under six years of age," Malaria Journal, vol. 7, article 41, 2008.

[84] D. J. Springer and V. Chaturvedi, "Projecting global occurrence of Cryptococcus gattii," Emerging Infectious Diseases, vol. 16, no. 1, pp. 14-20, 2010.

[85] P. G. Sohnle, B. L. Hahn, and V. Santhanagopalan, "Inhibition of Candida albicans growth by calprotectin in the absence of direct contact with the organisms," Journal of Infectious Diseases, vol. 174, no. 6, pp. 1369-1372, 1996.

[86] M. Bianchi, M. J. Niemiec, U. Siler, C. F. Urban, and J. Reichenbach, "Restoration of anti-Aspergillus defense by neutrophil extracellular traps in human chronic granulomatous disease after gene therapy is calprotectin-dependent," Journal of Allergy and Clinical Immunology, vol. 127, no. 5, pp. 12431252, 2011.

[87] V. Aimanianda, J. Bayry, S. Bozza et al., "Surface hydrophobin prevents immune recognition of airborne fungal spores," Nature, vol. 460, no. 7259, pp. 1117-1121, 2009.

[88] S. Bruns, O. Kniemeyer, M. Hasenberg et al., "Production of extracellular traps against Aspergillus fumigatus in vitro and in 
infected lung tissue is dependent on invading neutrophils and influenced by hydrophobin RodA," PLoS pathogens, vol. 6, no. 4, Article ID e1000873, 2010.

[89] T. Narasaraju, E. Yang, R. P. Samy et al., "Excessive neutrophils and neutrophil extracellular traps contribute to acute lung injury of influenza pneumonitis," American Journal of Pathology, vol. 179, no. 1, pp. 199-210, 2011.

[90] S. Molloy, "Bacterial pathogenesis: escaping the net," Nature Reviews Microbiology, vol. 4, pp. 242-246, 2006.

[91] L. Vitkov, M. Klappacher, M. Hannig, and W. D. Krautgartner, "Extracellular neutrophil traps in periodontitis," Journal of Periodontal Research, vol. 44, no. 5, pp. 664-672, 2009.

[92] T. A. Fuchs, A. Brill, D. Duerschmied et al., "Extracellular DNA traps promote thrombosis," Proceedings of the National Academy of Sciences of the United States of America, vol. 107, no. 36, pp. 15880-15885, 2010.

[93] Z. G. Fridlender, J. Sun, S. Kim et al., "Polarization of tumorassociated neutrophil phenotype by TGF- $\beta$ : "N1" versus "N2" TAN," Cancer Cell, vol. 16, no. 3, pp. 183-194, 2009.

[94] T. Lögters, S. Margraf, J. Altrichter et al., "The clinical value of neutrophil extracellular traps," Medical Microbiology and Immunology, vol. 198, no. 4, pp. 211-219, 2009.

[95] S. Margraf, T. Lögters, J. Reipen, J. Altrichter, M. Scholz, and J. Windolf, "Neutrophil-derived circulating free DNA (CFDNA/NETs): a potential prognostic marker for posttraumatic development of inflammatory second hit and sepsis," Shock, vol. 30, no. 4, pp. 352-358, 2008. 

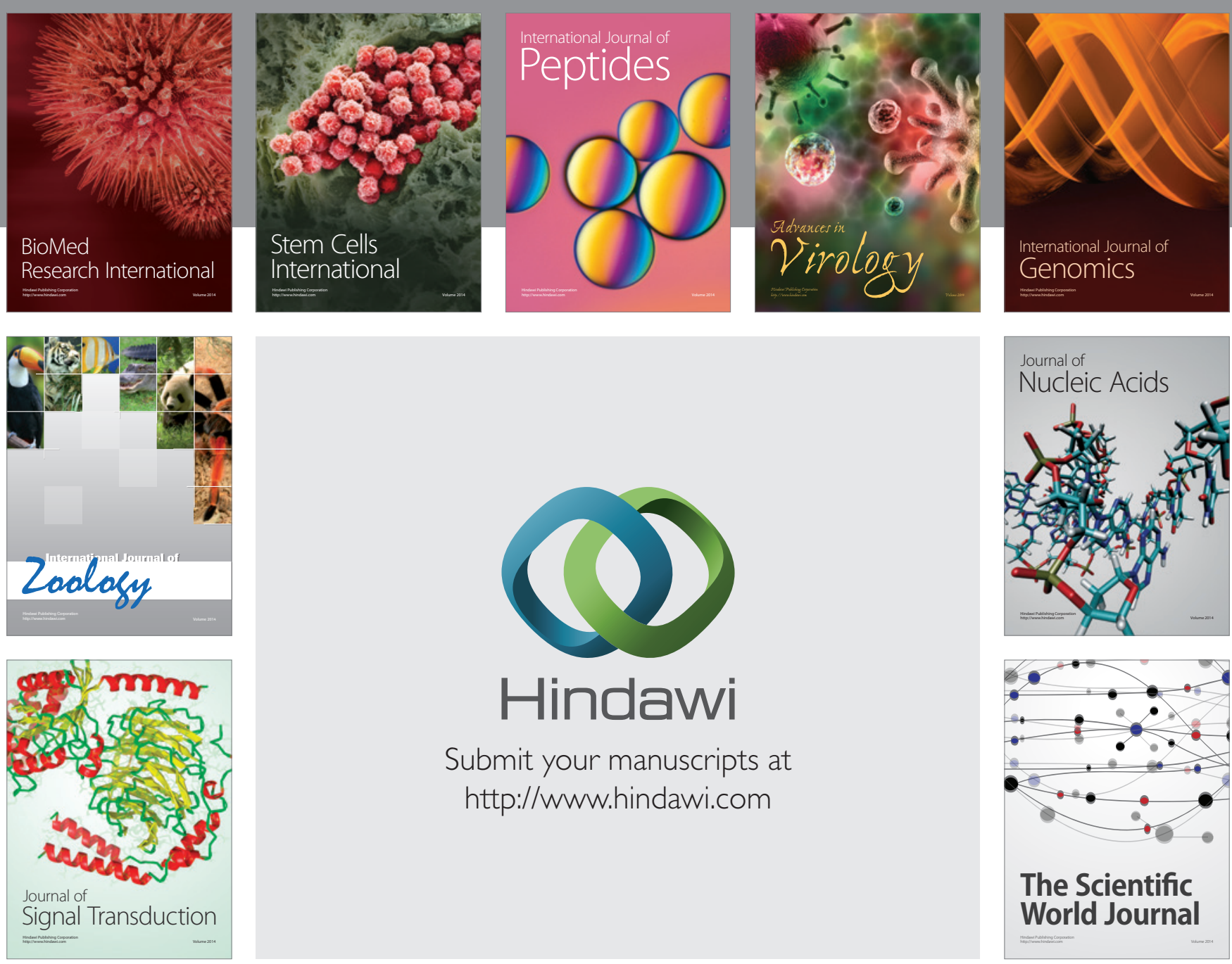

Submit your manuscripts at

http://www.hindawi.com
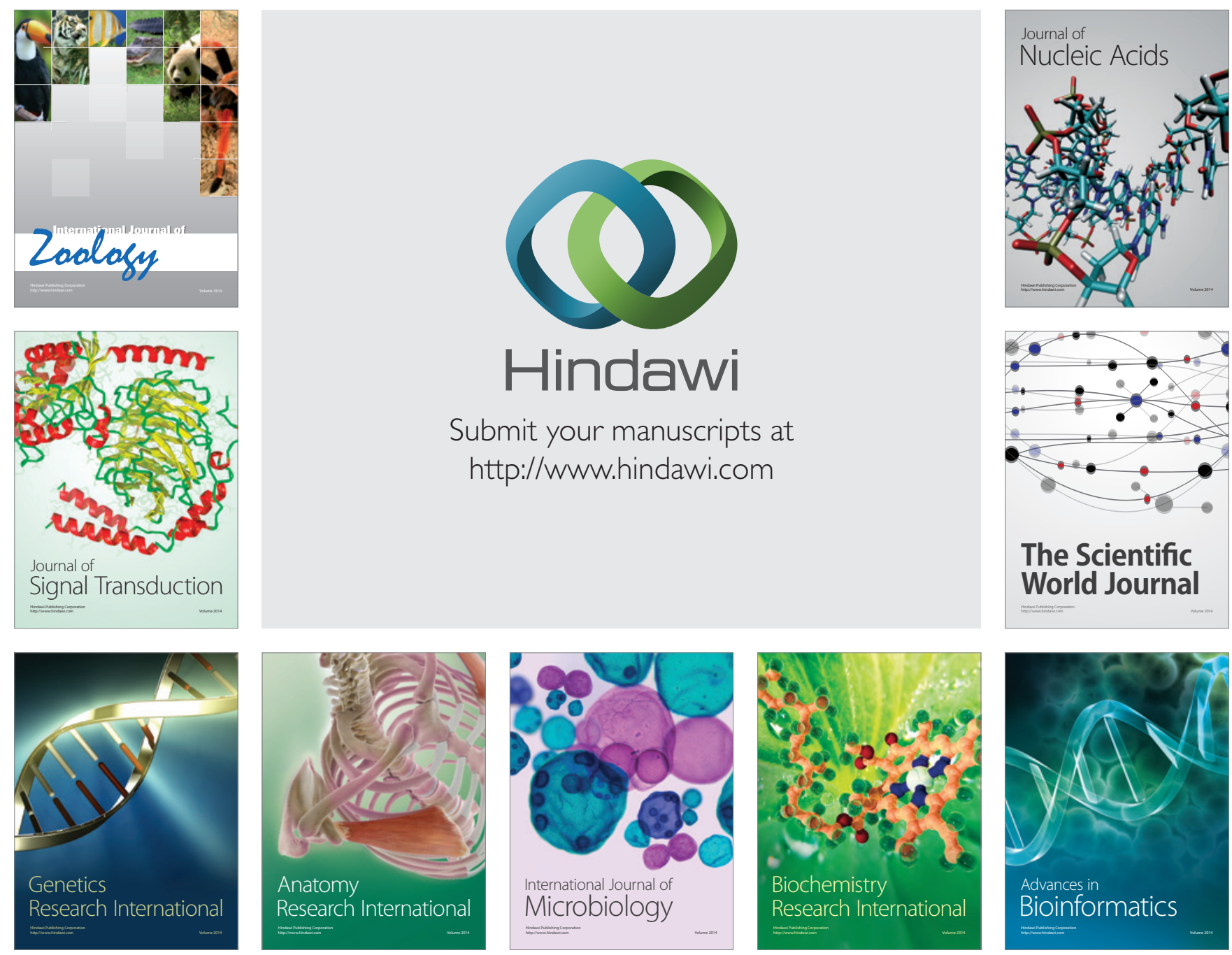

The Scientific World Journal
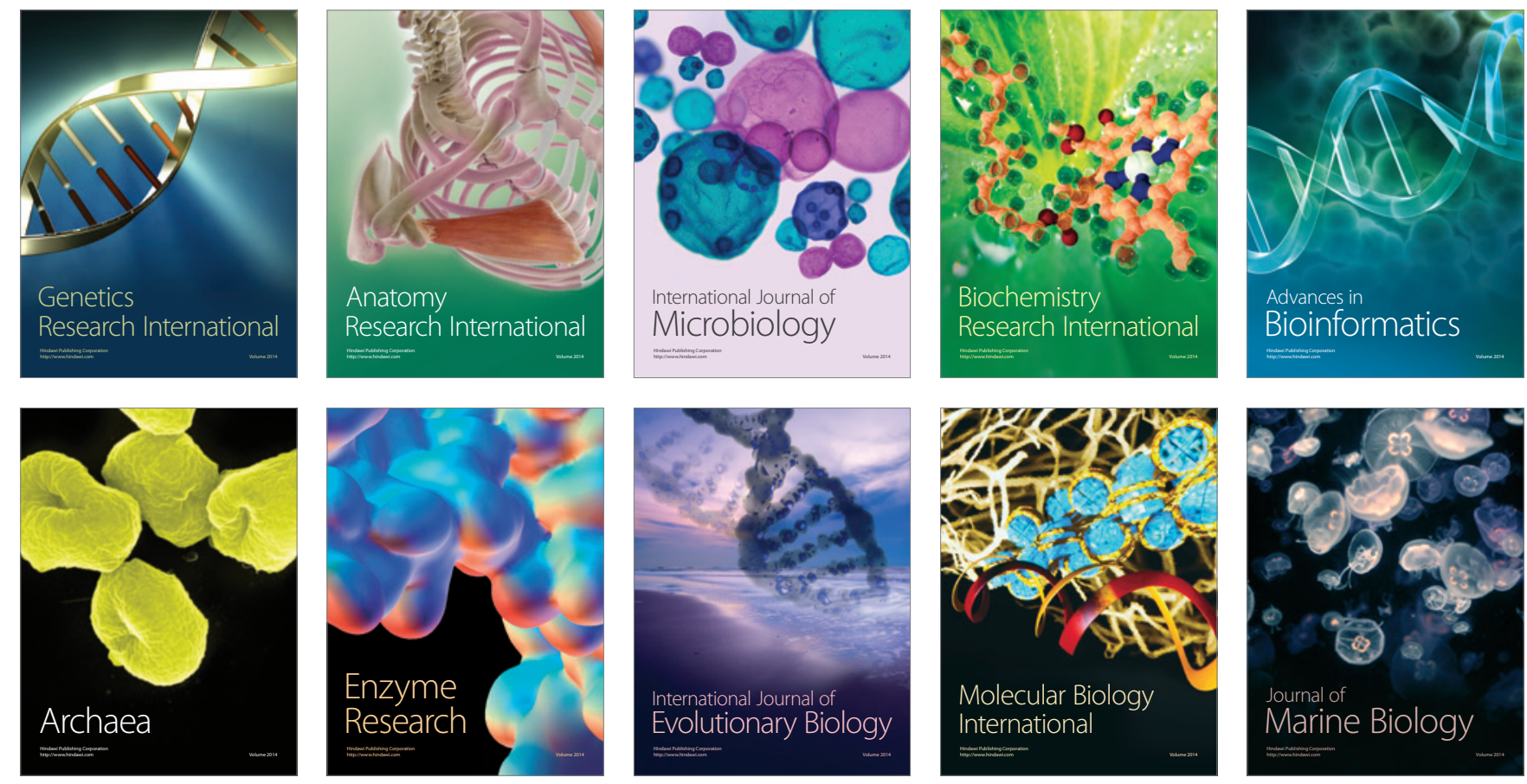